\title{
Research on EIoT Reservation Algorithm Based on Deep Learning
}

\author{
Jennefer Mononteliza1)
}

\begin{abstract}
Based on the resource slicing strategy of deep reinforcement learning, this paper proposes a method framework for emergency Internet of Things, No.EIoT) slice resource reservation and multi-heterogeneous slice resource sharing and isolation. In view of the differentiated service quality requirements of multiple network slices, and the different requirements of different slices for speed and delay indicators, a shape-based two-dimensional knapsack problem is used and heuristic algorithm is used to numerically solve it. Experimental results show that, compared with the traditional NVS, No.network virtualization substrate) and Netshare algorithms, the Dueling DQN algorithm is better, effectively balancing the performance of heterogeneous coexisting slices.
\end{abstract}

Keywords: EIoT, Reservation Algorithm, Deep Learning

\section{Introduction}

5G aims to provide thousands of times the transmission capacity increase of $4 \mathrm{G}$, the connection of at least 100 billion IoT devices, the transmission rate of up to 10Gbit/s, and the ultra-low latency user experience as low as milliseconds. In addition to human-to-human communication, the next-generation mobile Internet will realize zero-distance connections between humans and machines, and between machines and machines. Wireless technology will promote future economic and social development in a brand-new way. Therefore, ultra-low latency transmission is regarded as one of the main technical features of $5 \mathrm{G}$ systems, and its goal is to achieve an end-to-end transmission delay of less than 1ms, thereby supporting human-to-machine and machine-to-machine real-time communication and remote control applications. In addition to strict requirements in terms of transmission delay, reliability and throughput, 5G has also made major changes to the next-generation mobile Internet architecture. Software-defined networking and network function virtualization technologies, as innovative technologies for 5G network architecture, enable infrastructure networks to be divided into several logical networks, allowing multiple differentiated applications to share the same physical network and resources, the

Received(April 28, 2020), Review Result(1st: June 11, 2020, 2nd: July 31, 2020), Accepted(August 28, 2020)

1) (Professor) University of Antique, Antique, Philippines

email: jenneferjmonteliza@gmail.com 
so-called network slicing technology. Each independent slice can call the virtual network function running on the public network infrastructure, and configure and adjust its communication and computing resources as needed to meet the specific business requirements of specific network slice applications[1][2]. Usually, each tenant signs a service level agreement with the infrastructure provider. Therefore, through custom slicing applications and functions, and dynamic allocation of resources of custom slicing, public mobile networks can support specific emergency IoT slicing and ensure the coexistence and safe isolation of the slicing and other mobile network slicing[3][4]. To sum up, the application business requirements for emergency Internet of Things. First of all, need to ensure the quality of service of a single emergency Internet of Things, No.EIoT) slice, allowing tenants to manage the network performance of their customized slices. Secondly, they need to consider multiple differences. The problem of the coexistence of architecture slices is to realize the economies of scale of the infrastructure by reusing the slice traffic.

In recent years, there has been a lot of research work on resource slicing, but the following problems still exist in heterogeneous mixed data flow scenarios: 1) In the case of limited wireless resources, how to ensure the resource efficiency of all slices and accurately meet the requirements Slicing demand. 2) How to dynamically allocate resources for each slice according to the requirements of the service level agreement, No.SLA) to meet the quality of service, No.QoS, quality of service) requirements of different slices. 3) In a highly dynamic environment where the flow status changes in real time, how does the resource allocation plan intelligently respond to the changing characteristics of the network and adapt to changes? Based on the virtualization management program, this paper allocates resources for multiple virtual base stations hosted on different nodes, and schedules the corresponding hardware physical resources and wireless resources for them, so as to realize the sharing of spectrum resources and data reuse[5]. Among them, a physical RB, No.PRB, physical resource block) is allocated to different virtual base station nodes as a radio resource with the smallest granularity. The virtual base station is used to realize resource sharing among multiple network slices and an isolation mechanism based on traffic shaping[6]. Literature [7][8] applies deep reinforcement learning methods to the problem of multi-slice resource allocation. Literature [7] mainly focuses on mobile Internet of Vehicles content cache resources. Literature [8] only considers 2 slice examples, based on the traditional nature DQN, No.deep Q-network) algorithm. Based on the existing work in [8], this paper improves the Dueling network structure to accelerate learning convergence for the problem of autonomous resource allocation and customization of mixed traffic, and uses an adaptive linear reward mechanism to automatically balance the resource 
utilization and QoS satisfaction of slices. And verified the safety isolation effect.

This paper mainly studies a general slice resource reservation method for specific emergency IoT scenarios, and considers the dynamic security isolation of multi-slice performance in the coexistence of multiple heterogeneous slices. For specific emergency IoT, the resource reservation-based method can provide strict quality of service assurance, protection and isolation of resources between slices, and provide resource customization and stability. Therefore, for emergency IoT applications, this article mainly uses resource reservation to ensure end-to-end delay and reliability, and provides customized physical resources for its users, while promoting the coexistence of multiple heterogeneous slices.

\section{System Model}

\subsection{Network Model}

As shown in Figure 1, the multi-slice network architecture proposed in this article uses software defined network, No.SDN) and network function virtualization, No.NFV) network architectures. Specifically, it includes SDN controller, end user equipment, No.UE), network slicing, base station, and spectrum resources. The SDN controller is responsible for resource scheduling and decision-making at the slice level, and uses the message signaling interface to notify the specific base station to adjust the resource reservation or allocation amount of its slice. The base station provides a certain number of $\mathrm{RB}$ resources for different slices. The terminal user equipment sends a resource request by carrying the slice identification information to which it belongs, and obtains and occupies the $\mathrm{RB}$ resource of the slice to which it belongs from an associated base station. In terms of resources, this article mainly considers spectrum resources, that is, RBs composed of time domain and frequency domain. This article mainly considers 4 types of slices: high-definition video, No.HDTV), massive terminal Internet of Things, No.MIoT), EIoT, and UEb , No.UE broadband).

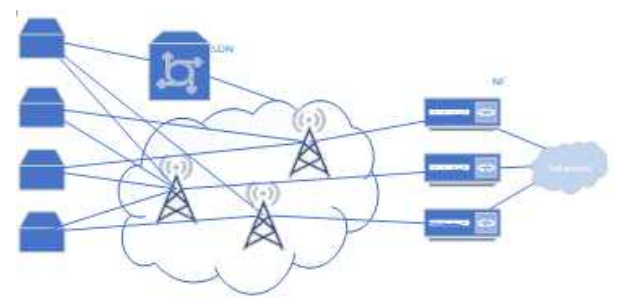

[Fig. 1] The multi-slice Network Architecture 
Aiming at the coexistence of multiple heterogeneous slices, this paper proposes a method framework for general slice resource management based on deep reinforcement learning, as shown in [Fig. 2]. The basic principle is: the DRL agent continuously interacts with the wireless network environment and obtains the current state of the environment, and the agent selects an action to execute according to the current environment state. After performing this action, the current state of the environment will be transferred to another state with a certain probability. At the same time, the environment feeds back to the agent a reward or punishment. The agent keeps repeating the above process to get as many rewards from the environment as possible.

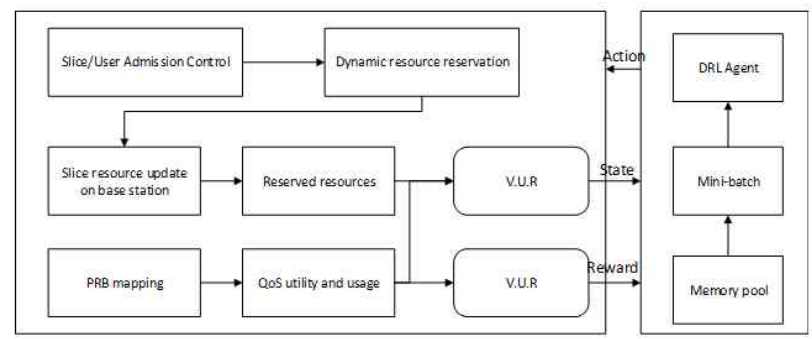

[Fig. 2] Framework of Resource Reservation Method Based on DRL

First, resource reservation is divided into two stages: initial resource reservation and dynamic resource reservation. Initial resource reservation means that each base station allocates a fixed resource ratio to each slice according to the signed service level agreement. Since the resources of the emergency IoT slice in a single base station are limited, the number of admitted users needs to be controlled. Generally, user admission control can be modeled as a 0-1 integer programming problem and solved by existing algorithms. Its goal is to maximize the number of Internet of Things terminals on the premise of ensuring the terminal's speed and delay requirements.

Second, due to the inherent characteristics of terminal mobility and the time-varying nature of the wireless environment, once the number of terminals in a single base station's emergency IoT slice changes, there may be insufficient resources or excess resources. Therefore, it is necessary to predict according to the load status of the emergency IoT, and dynamically adjust the sliced resources to adapt to the dynamic changes of the emergency IoT slice resource demand. Furthermore, the dynamic adjustment of slice resources is mapped to different base stations, that is, dynamic resource reservation.

Third, the user-level physical resource allocation is performed by the base station to map the connected user data to the PRB set. When the reserved resources V of a specific slice are 
mapped to base station resources, the base station needs to allocate PRB resources to connected terminals of this slice. However, different multi-user physical resource allocation methods will cause differences in system resource utilization $\mathrm{R}$ and user QoS satisfaction $\mathrm{U}$. On the premise of ensuring slice QoS satisfaction, how to maximize the resource efficiency of slices is a question of resource customization within a single slice.

Finally, the DRL agent completes a slice resource allocation, and the terminal can obtain the corresponding physical RB resources. Furthermore, the terminal obtains the QoS satisfaction evaluation, and obtains statistics on the resource utilization efficiency of the slice. Thereby, a reward or punishment is fed back to the agent, and the wireless network environment is transferred and updated to the next state. The agent composes the current environment state, resource allocation action, feedback reward, and the next state of the environment into a four-tuple, and stores it in the memory pool as a sample. Through the memory playback mechanism, the agent will randomly select mini-batch sample data from the memory pool according to the training cycle configuration to perform intensive training on the agent, thereby continuously updating the coefficients of the neural network to reduce loss.

\subsection{Delay Model}

For emergency IoT scenarios, EIoT slices have higher requirements for response delay, but may have lower requirements for speed. Different application service slices have different requirements for response delay and transmission rate. Therefore, a delay model is required to effectively evaluate the service delay of the emergency IoT base station to each end user. This article makes the following assumptions: 1) The arrival time of each data packet sent by the end user $\mathrm{u}$ obeys an exponential distribution, with an average value of $\frac{1}{\lambda} s$, and the arrival time interval of any two adjacent data packets is independent of each other, $\lambda u$ is the end user $u$ The data packet arrival rate, its unit is packets per second. 2) The data packet length of the terminal $\mathrm{u}$ served by a particular slice $\mathrm{s}$ is $L_{u k} b i t$, and the data packet sizes of different slice applications are independent of each other. Therefore, the time for terminal $\mathrm{u}$ to send a data packet to base station $\mathrm{k}$ is

$$
t_{u k}=\frac{L_{u}}{c_{u k}}=\frac{1}{c_{u k}^{*}} \#
$$

Among them, is the transmission rate actually obtained by the terminal $u$ from the base station $\mathrm{k}$, in bit/s; is the normalized actual transmission rate in packets per second. Based on 
the above assumptions, according to the $\mathrm{M} / \mathrm{M} / 1$ theoretical model of queuing theory[9], the average service delay of the data packet of user $u$ can be calculated as

$$
\gamma_{u k}=\frac{1}{\frac{1}{t_{u k}-\lambda_{u}}}=\frac{1}{a_{u k} \cdot c_{u k}^{*}-\lambda_{u}} \#
$$

Among them, $a_{u k}$ is the correlation variable between terminal $\mathrm{u}$ and base station $\mathrm{k}$. If user $\mathrm{u}$ is associated with base station $\mathrm{k}, a_{u k}$ is 1 ; otherwise, it is 0 .

\subsection{Utility Model}

The utility function is mainly used to characterize the terminal's satisfaction with the service quality. In addition, it is also part of the reward function that is fed back to the agent. It is not difficult to understand that different slices have different service types and have different requirements for rate or delay, that is, different slices have different satisfaction functions. For example, the satisfaction calculation of emergency IoT slicing mainly depends on time delay, while HDTV slicing mainly depends on transmission rate and so on. Assume that the minimum rate requirement of the terminal $u$ served by slice $s$ is $c_{u}^{\min }$, and the maximum delay requirement is $T d_{u}^{\mathrm{min}}$. In a scheduling period $\mathrm{T}$, each terminal automatically calculates the satisfaction of service quality[Sat] $u$ according to the obtained service rate and delay, and then averages the satisfaction of all terminals in the slice to obtain the slice user's satisfaction Average satisfaction function $S a t_{u}$.

Specifically, the rate-sensitive service quality satisfaction of terminal $u$ is

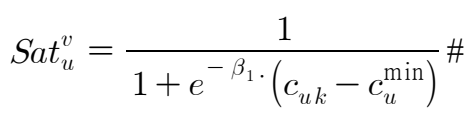

The terminal $u^{\prime}$ s satisfaction with the delay-sensitive service quality is

$$
S a t_{u}^{d}=\frac{1}{1+e^{-\beta_{2} \cdot}\left(T d_{u k}-T d_{u}^{\max }\right)} \#
$$

Among them, $\beta_{1}$ and $\beta_{2}$ are the slopes of the sigmoid function[10]. Therefore, through equations, No.3) and , No.4), the average satisfaction degree of slice s can be calculated as 


$$
S a t_{s}=\frac{1}{U_{s}} \cdot \sum_{u \in U_{s}} \frac{\left(S a t_{u}^{v}+S a t_{u}^{d}\right)}{2} \#
$$

Among them, $U_{s}$ represents the set of end users of slices.

\section{Problem Modeling}

\subsection{Slice Rsource Rrvation based on Dep Rinforcement Larning}

For emergency IoT slice resource reservation, the demand for slice resources needs to be dynamically predicted. This problem can be modeled as a Markov decision process and solved by deep reinforcement learning algorithms to realize resource sharing and isolation of multiple heterogeneous slices. Deep reinforcement learning provides a general algorithm framework for heterogeneous slice resource demand prediction and slice resource reservation, including the three basic elements of state space State, action space Action, and reward function Reward. For the emergency IoT scenario, the definition is as follows.

\subsubsection{State}

The emergency IoT status includes three aspects of information, namely the number of resources reserved for the current slice, the number of slice resources occupied, and the average service quality satisfaction of the slice. Specifically, it can be expressed by the following three numerical values: the resource reservation ratio $V_{s}$ of the slice refers to the proportion of the slice in the entire system resources, not the proportion of the resources on a single base station. The resource utilization rate of a slice $R U_{s}$. The resource utilization rate refers to the ratio of the resources actually used to the reserved resources of the slice. Slice QoS satisfaction $S a t_{s}$ refers to the average value of service quality satisfaction of all terminals in the slice. For the coexistence of multiple heterogeneous slices of emergency IoT, the State set is defined as $\left[V_{s}, R U_{s}, \quad U S a t_{s}\right]$.

\subsubsection{Action Represents the Set of Actions Performed}

Each time the DRL agent acquires a state, it will select and execute an action according to the greedy algorithm. Aiming at the problem of dynamic resource reservation between heterogeneous slices, the action operation is to dynamically adjust the system proportion of 
slice resources. In other words, increase or decrease a certain percentage on the basis of the original reserved resource quantity. Assuming that the resource ratio reserved for the initial slice is $V_{s}$, and the executed action is a, the adjusted resource ratio is $V_{s}^{\prime}=V_{s} \cdot$, No.1 $+a$ ). Since the DRL agent can only select actions in the discrete action space, it is necessary to use continuous rewards to represent the rewards returned by environmental interaction. In each iteration, the agent will select and perform an action based on the current environment state, and then the environment will move to the next state and give the agent a reward. Generally speaking, this reward should reflect whether the selected action is correct. For the multi-slice coexistence scenario of emergency IoT, the rewards should be discretized with the slice action space. If the dimension of the action space of a single-slice scene is $M$ and $N$ slices coexist in the scene, the dimension of the action space is MN. Therefore, for a scene where heterogeneous slices coexist, the degree of dispersion and granularity of the action space have a greater impact on the convergence speed.

\subsubsection{Reward, which means the Reward of Feedback from Environmental Interaction}

In each iteration, the agent will select and perform an action based on the current environment state, and then the environment will move to the next state and give the agent a reward. Generally speaking, this reward should reflect whether the selected action is correct. For the multi-slice coexistence scenario of emergency IoT, the rewards should be related to slice QoS satisfaction and slice resource utilization. Assuming that the slice QoS satisfaction is, and the slice resource utilization is, the reward function of a single slice is

$$
\gamma_{s}=\alpha \cdots a t_{s}+\beta \cdot R U_{s} \#
$$

Among them, $\left.\alpha, N_{0} .0 \leq \alpha \leq 1\right)$ is the weight of slice QoS satisfaction, and $\beta, N o .0 \leq \beta \leq 1)$ is the weight of slice resource utilization. The reward function of the entire system is defined as the sum of all slice reward functions. If $\alpha$ and $\beta$ introduce a linear relationship, that is, $\beta=1-\alpha$, an adaptive reward-return model can be defined, which can automatically adjust these two weight values and automatically balance the impact of two independent factors on rewards and returns[11]. Using a score-based merging mechanism, the reward model can automatically learn and adjust to adapt to new scenarios.

$$
\alpha=\sigma\left(S a t_{s}-R U_{s}\right) \#
$$


Among them, $\left.\sigma, N_{o}.\right)$ is the sigmoid function, $\left.\sigma(x)=\frac{1}{e^{-x}}, \alpha \in, N_{o} .0,1\right)$, indicating the importance of each reward metric. The Sigmoid function is often used as the activation function of neural networks to map variables to $0 \sim 1$. Since the satisfaction function and resource utilization rate are both 0 to 1 , the $\alpha$ in formula, No.7) is also 0 to 1 , so as to ensure that in formula, No.6) is 0 to 1 . The algorithm flow of automatic slicing resource reservation is as follows.

1) Initialize the memory pool capacity $D$ and the number of mini-batch samples $d$;

2) Initialize the dimensions of the input state and output action space, and initialize the neural network coefficients randomly;

3) Set the probability control parameter $\varepsilon$ of epsilon greedy algorithm

4) Select the action according to the current state $s$. The selection of the specific action adopts the epsilon strategy, that is, a value $\pi$ is randomly generated. If $\pi\langle\epsilon$, an action $\alpha$ is randomly selected from the output action set, otherwise the action $\alpha$ with the largest $Q$ value is selected;

5) Perform action $a$, that is, increase or decrease the proportion of slice resources in the system, and map the proportion of slice resources to the proportion of base station resources, and then generate system feedback through terminal physical resource allocation, that is, user QoS satisfaction and resource utilization, and Through formula , No.6) and formula , No.7), the reward return $\gamma_{s}$ is calculated;

6) Count the number/proportion of the resources of the slice on each base station, update the proportion of the slice in the system resources, and generate the next environment state $s^{\prime}$;

7) Store the quadruple tuple $\left\langle s, a, r, s^{\prime}\right\rangle$ as a new sample in the memory pool;

8) If the memory pool is full, randomly select a batch of data as mini-batch for neural network training;

9) If the index value of the current episode reaches the upper limit, the algorithm terminates, otherwise skip to 4). Episode represents the process from the beginning to the end of the reinforcement learning agent executing a strategy in the environment.

In the above process, step 5) adopts the definition of adaptive Reward function as the main innovation of this algorithm, which simplifies manual parameter configuration and can automatically complete parameter configuration. The Dueling network structure accelerates the algorithm convergence. This article is used in multi-slice coexistence scenarios. The performance of the automatic slicing resource reservation algorithm is verified. 


\subsection{Shape-based User Resource Customization}

Aiming at the coexistence of heterogeneous slices, this paper adopts a shape-based intra-slice physical resource allocation model. Similarly, literature [12] modeled the spectrum resource as a discrete two-dimensional time-frequency grid. By defining the utility function of service quality requirements, the physical $\mathrm{RB}$ allocation is modeled as a two-dimensional geometric knapsack problem. A heuristic algorithm is used to search and select different combinations of resource allocation options, and the performance of the algorithm is evaluated according to indicators such as transmission rate and service delay. This article mainly extends it to multiple heterogeneous slice scenarios. For emergency IoT and multi-slice coexistence application scenarios, assume that $\mathrm{K}$ base stations are deployed. For any base station $k \in\{1,2, \ldots, K\}, \mathrm{S}$ slices are deployed. For any slice $s \in\{1,2, \ldots, S\}$, each slice has different quality of service requirements. Terminals are evenly distributed around the base station, and any terminal $u \in\{1,2, \ldots, U\}$ may request the service of slice s. It is assumed that the service quality requirements of all terminals of the same slice service are the same, and the minimum rate requirement and the maximum delay requirement are recorded as $c_{u}^{\min }$ and $T d_{u}^{\max }$ respectively. For wireless access network spectrum resources.

Based on the above analysis, this paper proposes to calculate the time slot and frequency domain $\mathrm{RB}$ distribution shape required by the user to send the data stream according to the minimum transmission rate and maximum waiting delay requirements of the slice user, that is, the more refined QoS requirements, and the shape-based Physical resource allocation.

\section{Experimental Results}

\subsection{Experimental Configuration}

The scenario configuration of the system simulation in this paper refers to the relevant standards of the 5G access network, and the main system parameter configuration is shown in [Table 1]. 4 base stations are uniformly deployed within a range of $700 \mathrm{~m} * 700 \mathrm{~m}$, the coverage radius of the base station is $150 \mathrm{~m}$, and every two adjacent base stations maintain a fixed distance of $120 \mathrm{~m}$. For the wireless propagation environment, the path loss model adopted is 
Where $d$ represents the distance between the user and the base station, and $f$ is the channel frequency

In view of the coexistence of multiple heterogeneous slices, this paper defines 4 different types of slice instances. Each specific slice provides specific services and has different QoS requirements. 1) EIoT slice has the highest priority, its maximum delay requirement is $10 \mathrm{~ms}$, minimum rate requirement is $10 \mathrm{kbit} / \mathrm{s}$, data packet size is $120 \mathrm{bit}$, packet arrival rate is 100 packets/s[13]; 2) HDTV slice is the smallest The rate requirement is $500 \mathrm{kbit} / \mathrm{s}$, the maximum delay requirement is $120 \mathrm{~ms}$, and the data packet size is $4000 \mathrm{bit}[13]$; 3) The maximum latency requirement of MloT slice is $105 \mathrm{~ms}$, the minimum rate requirement is $12 \mathrm{kbit} / \mathrm{s}$, and the data packet size is 500 bit. The arrival of data packets obeys an exponential distribution, with an average of 100 pieces/s; 4) The minimum rate requirement of $\mathrm{UEb}$ slice is $100 \mathrm{kbit} / \mathrm{s}$, the maximum delay requirement is $100 \mathrm{~ms}$, and the data packet size is 400 bits. The simulation experiment lasts for $420 \mathrm{~s}$ in total, that is, the user continues to transmit data packets for $1000 \mathrm{~s}$. The data packets of the UEb slice and HDTV slice are modeled as exponentially distributed arrivals, with an average of 100 packets per second.

[Table 1] System Parameter Configuration

\begin{tabular}{|c|c|}
\hline Parameter & value \\
\hline Slice category & 4 \\
\hline Number of base stations/piece & 4 \\
\hline Number of users/one & $189 \sim 435$ \\
\hline System bandwidth/ MHz & 5 \\
\hline Base station transmit power/dBm & 30 \\
\hline Subframe length / ms & 1 \\
\hline Frame length / ms & 10 \\
\hline Number of base station sub-channels/ one & 25 \\
\hline User packet arrival rate/, No.packet·s-1) & [UEb:100,HDTV:100,MloT:100,EIoT:100] \\
\hline Minimum slicing rate requirement/, No.kbit·s-1) & [UEb:100,HDTV:500, MIoT:12, EloT:10] \\
\hline Maximum slicing delay requirement/ms & [UEb:100,HDTV:120,MloT:105,EIoT:10] \\
\hline Data packet size/bit & [UEb:400,HDTV:4000,MloT:500,EIoT:120] \\
\hline Data packet arrival method & EIoT: uniform distribution, others: exponential \\
& distribution \\
\hline Dynamic slicing cycle/ms & 200 \\
\hline
\end{tabular}

The algorithm parameters are configured as follows, the learning rate of the DRL algorithm 
is 0.01 , the epsilon-greedy value is 0.07 , the size of the memory pool is 8000 sample records, and each mini-batch contains 32 data record samples. Based on the existing literature research, the method in this paper is compared with the existing three benchmark methods, namely the aforementioned HapsliceR, NVS and Netshare.

\subsubsection{Q-learning}

The slicing strategy is based on reinforcement learning technology , No.Q-learning), which allocates resources to different slices with different needs and seeks the best solution. The slicing strategy estimates to provide resources for the slicing based on the traffic demand. However, resource slicing is done at the RB level, which makes the state space very large and leads to dimensionality disasters. Since Q-learning cannot solve complex machine learning problems, Q-table cannot converge, and Hap-SliceR uses Q-learning reinforcement learning technology, which cannot find the best solution for resource slicing problems of different types of traffic.

\subsubsection{NVS}

The global view setting is called static slice resource configuration, also called NVS. This solution assumes that the channel state of each user in the slice is known in advance, that is, re-association is not considered, the weight of each slice is considered, and the resources are statistically configured. Therefore, resource allocation is only based on the weight of the network slice.

$$
\omega_{s}=\sum_{u=1}^{U_{s}} R_{u} \forall u \in U_{s} \#
$$

Among them, $\omega_{s}$ is the weight of slice $s$ in the entire network, defined by the total data rate requirements of all its users; is the number of users of $\mathrm{s}$. According to the static slice resource configuration in [7], the fixed weight of the network resource sharing of this slice is

$$
W_{r a t_{s}}=\frac{w_{s}}{\sum_{s=1}^{S} \omega_{s}}, \quad \forall s \in\{1,2, \cdots, S\} \#
$$

Calculate the resource allocation of the slice through, that is, use equations, No.10) and, No.11) to determine the resource allocation between base stations. In NVS, the slice resource share is calculated by the proportion of the resource demand of the slice in the initial slice. NVS has at least two shortcomings: First, the total resource utilization of this cross-network 
slice is affected by the static resource reservation of each base station; second, NVS does not consider real-time and non-real-time traffic categories.

\subsubsection{NetShare}

NetShare believes that the resource portion of the slice has the largest and smallest resource limits at the system level. NetShare sets the upper and lower limits of base station-level resource allocation for each slice. It is assumed that all resources of a base station are completely allocated by all slices. According to the principle of proportional fairness, the dynamic resource allocation of slices can be periodically determined in NetShare by maximizing the utility function of the slice demand ratio scaled by the resource allocation ratio on the base station. The resources reserved by NetShare for a specific slice flow between all base stations

\subsubsection{Deep Q-Network}

Aiming at the problem of slicing and mode selection of cache resource in fog access network, a solution based on deep reinforcement learning is proposed. Reference [8] proposed a dynamic prediction algorithm for resource demand based on Deep Q-Network for the problem of heterogeneous slice wireless resource slice division, and used two slice examples to verify the beneficial effects. This paper is mainly based on the original work and expanded to 4 heterogeneous slice instances, and Deep Q-Network will be referred to as DQN algorithm in the following.

\subsection{Algorithm Convergence}

This section compares the convergence of DRL-based slice resource reservation algorithms. In the simulation experiment, 3000 episodes were run, each episode was 200ms long, points were taken for every 50 episodes and the Reward curve was drawn. The result is shown in Figure 4. The number of states of the Q-Learning algorithm is 128, and the Reward function is defined as shown in equation, No.13). $\beta$ represents the weight of resource utilization. In this paper, $\beta$ is set to 0 or 1 . When $\beta=1$, DuelingDQN and DQN start to converge from episode=500, and their system Reward reaches the maximum and is normalized to 0.95. Q-Learning starts to converge from episode $=2500$, and its system reward is 0.9 . When $\beta=0$, DuelingDQN and DQN also start to converge from episode $=500$, but the maximum system reward is 0.88 . Q-Learning starts to converge after episode $=2500$, and its maximum system Reward is 0.75 . The resource reservation algorithm based on DuelingDQN converges faster than 
DQN and Q-Learning algorithms.

\subsection{Comparison of Slice-level Resource Reservation}

Considering the coexistence of multiple heterogeneous slices, this section compares the slice resource allocation results based on Dueling DQN, DQN and Q-Learning. The resources reserved by the slice, the resources allocated by the slice are Allocated, and the resources actually used by the slice are used. Generally, the result of reserved resources is often greater than the result of actual allocated resources. If the number of users of a certain slice increases, the remaining unused resources can be reallocated to other slices, thereby ensuring safe isolation between slices. Compared with Q-Learning and DQN, the resource demand prediction and reservation results based on Dueling DQN are more accurate and reliable, and save resources, that is, it can accurately meet the differentiated needs of heterogeneous slice users with the least resources.

\subsection{Slicing Performance Comparison}

Generally, different slice resource allocations result in different slice satisfaction and slice resource utilization performance. This section compares with DRL, NVS and NetShare to evaluate the performance of DRL, No.including DQN and Dueling DQN) slice resource allocation proposed in this paper. The experimental results show that DuelingDQN can ensure that the resource utilization of the 4 slices is maintained at an acceptable level. It can be explained that when multiple heterogeneous slices coexist, the resource allocation method based on Dueling DQN has the best performance and can automatically balance the trade-off between slice satisfaction and resource utilization.

\section{Conclusion}

Aiming at the problem of intelligent scheduling and allocation of emergency IoT slice resources, this paper proposes a resource reservation based on deep reinforcement learning and a dynamic adjustment strategy of the resource ratio between slices to maximize the resource utilization of each slice on the premise of ensuring slice QoS satisfaction. And to ensure the performance safety isolation between slices. In response to the differentiated service quality requirements of heterogeneous slices, the physical resource customization problem is modeled as a two-dimensional knapsack problem, which is solved using the BLP algorithm to minimize 
the waste of resources. System simulation shows that the performance of DRL-based resource reservation strategy is superior to NVS and Netshare.

\section{References}

[1] X. Foukas, Network slicing in 5G: survey and challenges, IEEECommunications Magazine, (2017), Vol.55, No.5, pp.80-87.

[2] A. Kaloxylos, A survey and an analysis of network slicing in 5Gnetworks, IEEE Communications Standards Magazine, (2018), Vol.2, No.1, pp.60-65.

[3] F. Z. Yousaf, V. Sciancalepore, M. Liebsch, X. Costaperez, MANOaaS: A Multi-Tenant NFV MANO for 5G NetworkSlices, IEEE Communications Magazine, (2019), Vol.57, No.5, pp.103-109.

[4] Costa-Perez X., Swetina J., Guo T., Radio access networkvirtualization for future mobile carrier networks, IEEECommunications Magazine, (2013), Vol.51, No.7, pp.27-35.

[5] Zaki Y., Zhao L., Goerg C., LTE wireless virtualization andspectrum management, Third Joint IFIP Wireless and Mobile Networking Conference, (2010), October 13-15; Budapest, Hungary.

[6] Bhanage G., Seskar I., Mahindra R., Virtual base station:architecture for an open shared WiMAX framework, The Second ACM SIGCOMM Workshop on Virtualized Infrastructure Systems and Architectures, (2010), September 3; New Delhi, India, pp.1-10.

[7] Xiang H., Yan S., Andpeng M. A., realization of fog-RAN slicing viadeep reinforcement learning, IEEE Transactions on Wireless Communications, (2020), Vol.19, No.4, pp.2515-2527.

[8] Sun G., Gebrekidan Z. T., Boateng G. O., Dynamicreservation and deep reinforcement learning based autonomousresource slicing for virtualized radio access networks, IEEE Access, (2019), Vol.7, No.1, pp.45758-45772.

[9] Ross S., Introduction to Probability Models, 11th ed, Netherlands: Elsevier, (2014)

[10] Tang L., Zhang Y., Liang R., Virtual resource allocation algorithm for network utility maximization based on network slicing, Journal of Electronics \& Information Technology, (2017), Vol.39, No.8, pp.1812-1818.

[11] Liu F., Ren X., Liu Y., simNet: stepwise image-topic merging network for generating detailed and comprehensive imagecaptions, 2018 Conference on Empirical Methods in Natural Language Processing, (2018), November 2-4; Brussels, Belgium, pp.137-149

[12] Ginzalez A., Kuehlmorgen S., Festag A., Resource allocation for block-based multi-carrier systems considering QoS requirements, 2017 IEEE Global Communications Conference, (2017), December 4-8; Marina Bay Sands Expo and Convention Centre, Singapore, pp.1-7.

[13] Zeng Zhaomin, Wang Jihong, Guan Weili, A Deterministic Algorithm for Solving the Problem of Two-Dimensional Sheet Cutting Stock, Journal of Graphics, (2016), Vol.37, No.4, pp.471-475, DOI: 11996/JG.j.2095-302X.2016040471 